\title{
Taking the measure of quantum dynamics
}

1. Quantum Zeno effect and the many-body entanglement transition Authors: Y. Li, X. Chen, and M.P. A. Fisher

Phys. Rev. B 98, 205136 (2018)

2. Measurement-Induced Phase Transitions in the Dynamics of Entanglement Authors: B. Skinner, J. Ruhman, and A. Nahum

Phys. Rev. X 9, 031009 (2019)

3. Unitary-projective entanglement dynamics

Authors: A. Chan, R.M. Nandkishore, M. Pretko, and G. Smith

Phys. Rev. B 99, 224307 (2019)

4. Quantum Error Correction in Scrambling Dynamics and Measurement-Induced Phase Transition

Authors: S. Choi, Y. Bao, X.-L. Qi, and E. Altman

Phys. Rev. Lett. 125, 030505 (2020)

5. Dynamical Purification Phase Transition Induced by Quantum Measurements

Authors: M.J. Gullans and D.A. Huse

Phys. Rev. X 10, 041020 (2020)

6. Measurement-induced criticality in random quantum circuits

Authors: C.-M. Jian, Y.-Z. You, R. Vasseur, and A.W.W. Ludwig

Phys. Rev. B 101, 104302 (2020)

Recommended with a Commentary by S.A. Parameswaran, Rudolf

Peierls Centre for Theoretical Physics, University of Oxford

The past decade has seen growing interest in identifying universal aspects of non-equilibrium dynamics in quantum many-body systems. Originally, this was motivated by the success of cold-atom experiments in creating isolated systems evolving under simple local Hamiltonians. A key theoretical question is to explain how such isolated systems come to equilibrium under their intrinsic unitary dynamics where the system is always in a pure state. This is now understood as a process of 'scrambling': over time, unitary evolution encodes any local non-thermal information about the initial state of the system into the global structure of the quantum pure state. Such global information is inaccessible to local observables, since degrees of freedom in any subsystem are entangled with those in the remainder of the system. 

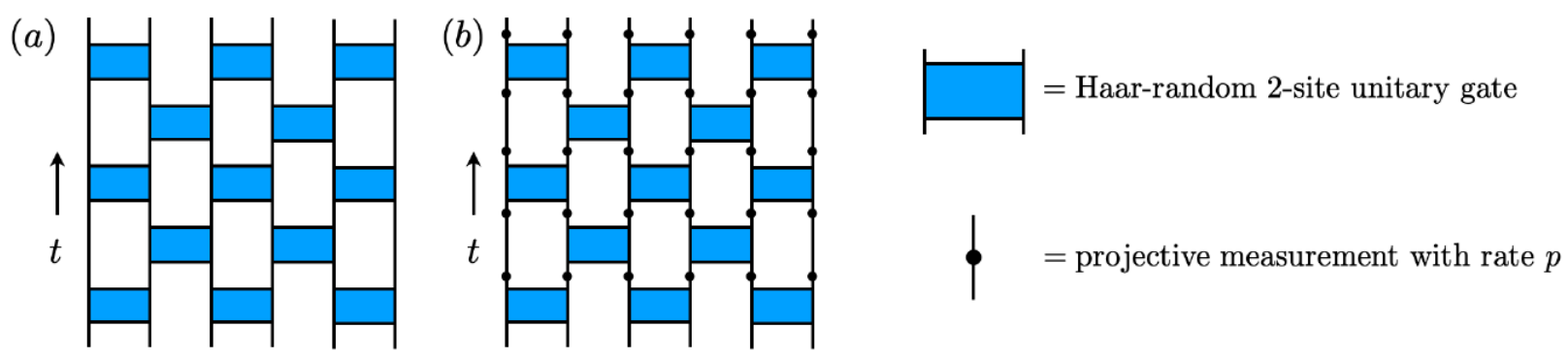

Figure 1: (a) Random unitary circuit (b) 'Hybrid' unitary-projective circuit.

A class of 'random unitary circuit' (RUC) models have proven especially useful in understanding essential aspects of such scrambling. In the simplest RUCs, time evolution is described by a 'brick wall' circuit (Fig. 1a) in which each gate is a randomly chosen unitary operator. In the spirit of random matrix theory, such models strip away inessential details of the system except for (in this example) locality and unitarity, even going so far as to discard deterministic evolution in the temporally random case. RUCs, and their modifications to include conservation laws or to describe time-periodic (Floquet) evolution, have yielded many insights into many-body chaos (see JCCM articles in October 2016 and June 2018), and are now part of the standard repertoire of the professional quantum dynamicist.

In many ways, RUCs are also toy models of the 'noisy intermediate-scale quantum' (NISQ) devices that are the current state of the art in quantum computing laboratories. As David DiVincenzo noted in this Journal Club ( $A$ Big Quantum Computer, JCCM October 2019), a condensed matter physicist might profitably view a NISQ device as an experimental platform offering unprecedented 'many-terminal' control of several strongly interacting quantum bits, with rather well-characterized interactions with their environment. As such, one might expect that it could play a similar role in exploring non-equilibrium quantum phases of matter as did the two-dimensional electron gas for their equilibrium counterparts.

However, a NISQ device has features not captured by purely unitary evolution. Quantum information is typically lost when an evolving system interacts with its environment, or is converted to classical information when measurements are performed and the results recorded. Joint unitary time evolution of system and environment together is generically non-unitary when viewed from the perspective of the system alone. Quantum information theorists often describe the evolution of such open quantum systems in terms of a quantum channel: a completely-positive, trace-preserving map $\mathcal{M}$ acting on the system density matrix, which can be written in terms of Kraus operators $K_{i}$,

$$
\mathcal{M}[\rho]=\sum_{i=1}^{k} K_{i} \rho K_{i}^{\dagger}, \quad \text { with } \quad \sum_{i=1}^{k} K_{i}^{\dagger} K_{i}=1,
$$

where each $K_{i}$ describes a distinct interaction between system and environment, and the label $i_{j}$ denotes the information recorded by the latter about the former. Repeated application of the channel defines a discrete time evolution of the system density matrix: after $t$ time steps, an initial density matrix $\rho_{0}$ evolves to $\rho_{t}=\sum_{i_{1}=1}^{k} \ldots \sum_{i_{t}=1}^{k} K_{i_{t}} \ldots K_{i_{1}} \rho_{0} K_{i_{1}}^{\dagger} \ldots K_{i_{t}}^{\dagger}$. We can view this as a sum over 'quantum trajectories' $K_{\boldsymbol{i}} \equiv K_{i_{t}} \ldots K_{i_{1}}$, labeled by the distinct ways $\boldsymbol{i}=\left\{i_{1}, i_{2}, \ldots, i_{t}\right\}$ in which the environment could have interacted with the 
system. To understand the meaning of a quantum trajectory, we specialize to the case where $K_{i}$ implements a projective measurement with outcome $i$, begin with an initial pure state $\rho_{0}=|\psi\rangle\langle\psi|$, and rewrite $\rho_{t}$ as an ensemble of pure states:

$$
\rho_{t}=\sum_{i} P(\boldsymbol{i}) \rho_{\boldsymbol{i}}, \quad \text { with } \rho_{\boldsymbol{i}}=\frac{K_{\boldsymbol{i}}|\psi\rangle\langle\psi| K_{\boldsymbol{i}}^{\dagger}}{\left\langle\psi\left|K_{\boldsymbol{i}}^{\dagger} K_{\boldsymbol{i}}\right| \psi\right\rangle} \text { and } P(\boldsymbol{i})=\left\langle\psi\left|K_{\boldsymbol{i}}^{\dagger} K_{\boldsymbol{i}}\right| \psi\right\rangle \text {. }
$$

Each trajectory thus yields a normalized pure state $K_{\boldsymbol{i}}|\psi\rangle / \| K_{\boldsymbol{i}}|\psi\rangle \|$ with a probability given by the Born rule for the sequence of measurement outcomes $\boldsymbol{i}$. One can view the measurements in two ways: as true measurements, recorded by an experimentalist, in which case the pure state generated by a trajectory is physically relevant, and can be accessed by 'postselecting' the appropriate measurement outcomes; or, as a fictitious mathematical device to map mixed state dynamics to an average over pure states.

The highlighted papers focus on 'hybrid circuits' of the form shown in Fig. 1b, where $\mathrm{RU}$ evolution is punctuated by projective measurements of qubits with rate $p$. Such circuits are more realistic models of NISQ devices with the simplest possible, measurement-only deviations from unitary dynamics. They focus on a class of questions that can be motivated as follows. If measurement outcomes are discarded, at late times the combined unitaryprojective evolution produces for any $p>0$ an 'averaged' density matrix, a thermally mixed state of the system entangled with its environment. Now, suppose instead that the environment is 'monitored' by recording the outcomes. This produces, as discussed above, an ensemble of pure states that appear with probabilities given by the Born rule. We can now reverse the order of the ensemble average and the entanglement calculation: first compute the entanglement of the pure state generated by an individual trajectory and then either average over trajectories using Born probabilities or post-select on the outcomes to consider a single trajectory or subset of trajectories. In the absence of measurements $(p=0)$, evidently there is a single trajectory producing a volume-law entangled state due to scrambling. When measurements are included $(p>0)$ and their outcomes recorded, how does the entanglement of pure states in the 'trajectory ensemble' behave? Does it remain volume-law, or do projective measurements arrest unitary entanglement growth? If the latter, does any non-zero measurement rate destroy volume-law behaviour, or is it robust up to some critical rate $p_{c}$ ?

The first three recommended papers, which appeared more or less simultaneously, explore the question of whether such an entanglement transition exists: the first two papers proposed and found a transition, while the third paper concurred while raising a qualitative argument for why such a transition might be prima facie surprising. This puzzle was resolved by the fourth recommended paper, which linked the transition to a change in the quantum errorcorrecting properties of the hybrid circuit, a theme expanded upon in the fifth paper that connected it to the ability of the hybrid circuit to 'purify' initially mixed states. The sixth paper (and parallel work by the authors of the fourth), introduced replica techniques that map the problem to a classical statistical mechanical model, allowing them to apply the methods of conformal field theory to address critical properties of the transition.

The paper by Li et al primarily studies a class of 'Clifford' circuits built from commuting stabilizer operators. This allows efficient simulation of large systems $\left(N \gtrsim 2^{9}\right.$ qubits), which would be unfeasible for a generic circuit. The authors used such simulations to identify two distinct phases: a 'weak measurement' phase that retains the late-time volume-law entangle- 
ment of the measurement-free evolution, and a 'strong measurement' or phase characterized by area law entanglement, where measurements prevent the growth of entanglement, analogous to the 'quantum Zeno effect'. They also began to explore properties of both phases and the transition within the Clifford setting. They also suggest that many features persist away from the Clifford limit, both by reproducing the two phases in small $(N=16)$ Haar-random circuits and by arguing that both Haar-random and Clifford circuits are unitary 2-designs, i.e. the averages of simple observables over both these types of random circuits approximates those over the full unitary group on $n$ qubits, $U\left(2^{n}\right)$.

Skinner et al take a complementary perspective, studying generic circuits with a simplified entanglement measure: the 'Hartley' or zeroth Renyi entropy $S_{A}^{(0)}$, which is related to the representability of the state as a tensor-network. Their analysis mapped the area-vs.-volumelaw question to one of distinct late-time scalings of the growth of entanglement between two halves of an infinite system, i.e. of saturation $\left(S_{A}^{(0)}(t) \sim t^{0}\right)$ vs. ballistic growth $\left(S_{A}^{(0)}(t) \sim t\right)$. Since it weights all eigenvalues of $\rho_{A}$ equally, $S_{A}^{(0)}$ is viewed as somewhat unphysical. However, its utility is that it is exactly computable: it saturates an upper bound on $S_{A}^{(n)}$ in terms of the minimal number of cuts required to disconnect the degrees of freedom in $A$ from those in $\bar{A}$ in the spacetime circuit (cf. Fig. 1b) representing the time evolution of the system. Measurements change this 'minimal cut' problem by breaking bonds, so that it now maps to 'first passage percolation': finding an optimum path in a disordered medium. This approach reveals a sharp transition: at low $p$, broken bonds are sufficiently rare that the minimal cut crosses $O(t)$ unbroken bonds, so $S_{A}^{(0)}(t) \sim t$; for $p>p_{c}$, broken bonds percolate, the minimal cut lies largely in the broken region, and $S_{A}^{(0)}(t) \sim t^{0}$. The percolation picture strongly suggests that $p_{c}$ is indeed finite, and that a robust volume-law phase survives a finite measurement rate in generic circuits. The authors also discuss critical scaling properties, and reason that the results for $S_{A}^{(0)}$ should be largely immune to details of trajectory averaging or post-selection. Finally, they numerically confirm that a transition also occurs in 'more physical' $n>1$ Renyi entropies, though with a distinct $p_{c}$, and explore critical scaling.

Notably, both the first two papers give an intriguing answer to the final question posed above: weak measurements appear insufficient to suppress a chaotic volume law. In the third recommended paper, Chan et al eventually also identify two distinct phases as a function of the measurement rate. However, en route to doing so, they flag (especially in an early preprint) a puzzling paradox: local unitary dynamics can only entangle a region $A$ with the rest of the system at its boundaries, while measurements can act anywhere inside $A$. So it appears that hybrid circuits can only inject an area-law's worth of entanglement in their unitary step, while removing a volume-law amount through measurement. From this logic, it seems that the volume law phase must always lose this 'battle of rates', and therefore that the critical strength for area law onset is at $p_{c}=0$, corresponding to arbitrary weak measurement. This is indeed seen in toy models of free fermions [1] and 'Bell-pair' dynamics, but is at odds with the existence of a robust weak-measurement volume-law phase in Clifford circuits and with the percolation picture. While Chan et al conclude that the former examples are special cases, the paradox nevertheless challenges the sort of intuitive scaling arguments beloved of physicists suspicious of overly formal computations.

An elegant resolution to this puzzle is provided in the paper by Choi et al, who linked the transition to a change in the quantum error-correcting properties of the circuit. They 
consider a simple toy model where two $N$-qubit systems $A$ and $B$ are entangled by sharing $\gamma N$ Bell pairs, and studied how measuring $p N$ qubits in $A$ changes the entanglement. For $N \gg 1$, by improving a bound provided by the decoupling theorem of quantum information they are able to show that the measured qubits in $A$ are effectively decoupled from those in $B$ as long as $p<1-\gamma$; measuring a finite fraction of qubits does not affect the entanglement between $A$ and $B$ as long as it lies below this threshold. They make the crucial point that the robustness of the volume-law phase lies in the 'hiding' of information by unitary dynamics in a manner inaccessible even to an extensive set of projective measurements, a feature of good quantum error correcting codes. They conjecture that the transition also marks a qualitative change in the ability of the circuit to reliably transmit quantum information.

This theme was picked up by the fifth recommended paper by Gullans and Huse who, instead of taking the initial state to be pure, asked whether evolution under the hybrid circuit is purifying, i.e. drives an initially mixed density matrix into a pure state. The intuitive picture is that as outcomes are monitored, there are increasingly fewer accessible pure states consistent with the measurement record and intermediate dynamics, leading eventually to purification. However, as they note, this naive expectation is contradicted by the existence of quantum error-correcting codes protected against extensive measurements, suggesting the existence of a 'purification transition' as a function of measurement rate. They accordingly map the volume-to-area law entanglement transition to one from a "mixed" phase where the purification time diverges exponentially with system size, to a "pure" phase with a constant purification rate in the thermodynamic limit. Consequently, at late times, an initially maximally mixed state has a nonzero entropy in the former phase, but becomes pure, and area-law entangled, in the latter. They then generalize the idea of a purification transition to a transition in the quantum channel capacity of the open-system dynamics, which quantifies its ability to transmit quantum information in an error-corrected fashion. In this setting, the critical measurement rate is a 'threshold' above which the channel no longer serves as a reliable conduit for quantum information. Simultaneously, Choi et al extended their results to prove a direct relationship between a hybrid circuit's steady-state entanglement entropy production and its channel capacity, offering a complementary perspective on the latter.

As two robust phases are seen to exist, a natural next step is to study the transition between them - the focus of the final recommended paper (and also the first paper in [4], which appeared simultaneously). Skinner et al draw parallels between the measurement transition and previous work on random tensor networks [2] by authors of the final recommended paper. As Jian et al show, replica techniques used to study random tensor networks can be fruitfully applied to the measurement transition, allowing it to be mapped to an effective classical statistical mechanical model. The calculation of a Renyi entropy $S_{A}^{(n)}$ becomes one of determining the free energy cost of a domain wall in the model associated with changing the boundary conditions in the region of $A$. Changing $p$ tunes the effective temperature of the classical model, driving it from an ordered phase at small $p$ where domain walls are costly leading to a volume law $\left(S_{A} \sim L_{A}\right)$, to a disordered one at large $p$ where domain walls are cheap and proliferate, corresponding to an area law $\left(S_{A} \sim L_{A}^{0}\right)$. This calculation becomes especially tractable in the limit of infinite-dimensional on-site Hilbert space, where it maps to a problem of 2D percolation as in the model studied by Skinner et al. More importantly, the mapping provides a crisp argument that the measurement-induced transition is controlled by a conformal field theory - albeit a non-unitary one - and there- 
fore has a dynamical critical exponent $z=1$, i.e. length and time scale similarly. Similar results were reached in parallel by Bao et al [4], who introduced a different replica scheme involving ancilla qubits coupled to a measuring device, and suggested a distinct probe of the transition in terms of 'Fisher information'. They also clarified that seeing a transition in the entanglement entropy requires a perfect measurement record: it is lost for any non-vanishing probability of unregistered measurements.

Besides the error-correction picture, there are other perspectives on the 'meaning' of the entanglement transition that are useful to note. The viewpoint used for most of this commentary most closely matches the situation in which the experiment actually records all outcomes that label a trajectory, in which case one can directly diagnose the transition by computing entanglement. Even if all outcomes are recorded, one could instead use them in conjunction with a classical computer and details of the unitary evolution to infer the state prepared by the circuit. Yet another picture is that the outcomes are not recorded, and the trajectories are an artifice introduced to simulate open-system evolution. As noted by Skinner et al, in the latter two settings, the transition is marked by the emergent intractability of identifying the prepared state or simulating the evolution as we enter the volume law phase.

Since these initial papers, a flurry of follow-on studies have explored diverse aspects of hybrid measurement-projective dynamics. These include the proposal of new dynamical phases enabled by measurement [3]; further explorations of both phases and criticality [4]; mappings to free theories [5] and mean-field-like approaches [6]; studies of other non-unitary evolutions e.g. in non-Hermitian systems [7], measurement-only protocols [8], or 'spacetime duals' [9] of unitary circuits; and a deepening of the links to error correction and channel capacity [10]. However, many of these proposals involve significant experimental overhead, such as post-selection, making them challenging to implement.

It is useful to step back and consider broader ramifications of this line of investigation. The perspective it gives on open quantum systems is rather distinct from traditional Lindblad dynamics, illustrating that NISQ devices, by enabling direct access to quantum trajectories, force us to consider open-system evolution in a new light. The prospect of using post-selection to prepare unconventional entangled steady states is also a promising medium-term goal, as is the broader question of understanding and classifying the possibilities enabled by such approaches. The practical utility of the error-correcting properties of hybrid circuits, and in particular, the extent to which there are efficient approaches to using them to encode and decode quantum information, remain largely unexplored. Attempts to clarify this have stimulated the application of statistical mechanical ideas to the study of quantum error correcting codes. Such approaches have been historically fruitful, for instance in the practical problem of distinguishing typical and worst-case computational complexity. It is possible that by sharpening the understanding of the interplay of locality, error correction, and noise, these techniques may play a similarly useful role in understanding the limitations and possibilities of near-term quantum technologies and the phase structure of non-equilibrium quantum matter. In a sense, the surge of theoretical work has provided an embarrassment of answers; the pace of experimental progress suggests that it may not be long before appropriate questions emerge. 


\section{References}

[1] X. Cao et al SciPost Phys. 7, 024 (2019).

[2] R. Vasseur et al, Phys. Rev. B 100, 134203 (2019).

[3] A. Lavasani et al Nat. Phys. 17, 342 (2021); S. Sang and T.H. Hsieh, arXiv:2004.09509; Y. Bao et al, arXiv:2102.09164.

[4] Y. Bao et al, Phys. Rev. B 101, 104301 (2020); Y. Li et al, Phys. Rev. B 100, 134306 (2019); A. Zabalo et al, Phys. Rev. B 101, 060301 (2020); R. Fan et al arXiv:2002.12385; Y. Li et al, arXiv:2003.12721; S. Vijay, arXiv:2005.03052.

[5] A Nahum and B. Skinner Phys. Rev. Research 2,023288 (2020), X. Chen et al,ibid 033017 (2020); C.-M. Jian et al arXiv:2012.04666

[6] J. Lopez-Piquerres et al, Phys. Rev. B 102, 064202 (2020); A. Nahum et al, Phys. Rev. X Quantum, 2, 010352 (2021).

[7] S. Gopalakrishnan and M. Gullans, arXiv:2012.01435. This work includes a lucid discussion of channels, trajectories, Lindblad dynamics, and measurement transitions.

[8] M. Ippoliti et al, Phys. Rev. X 11, 011030 (2021).

[9] M. Ippoliti and V. Khemani, Phys. Rev. Lett. 126, 060501 (2021); M. Ippoliti et al, arXiv:2103.06873; J. Napp et al, arXiv:2001.00021.

[10] M. Gullans and D. Huse, Phys. Rev. Lett. 125, 070606 (2020); M. Gullans et al arXiv:2010.09775; Y. Li et al, Phys. Rev. B 103, 104306 (2021). 